\title{
Brote de fungemias por Sarocladium kiliense en ocho hospitales públicos por contaminación intrínseca de ondansetrón intravenoso
}

\author{
Mauro Orsini', Fernando Otaíza', Pablo Vega², Luz María Hederra³, Paola Pidal', \\ Valentina Salas 5 , Paulina Coria ${ }^{6}$, Claudia Urízar ${ }^{6}$, Dino Sepúlveda², Margarita Palma², \\ Jorge Fernández ${ }^{7}$ M. Cristina Martínez ${ }^{8}$ y J. Carlos Hormazába/9
}

\section{Outbreak of fungemias by Sarocladium kiliense in eight public hospitals per intrinsic contamination of ondansetron intravenous}

Sarocladium kiliense is a saprophyte fungus that can cause opportunistic infections associated to invasive procedures. We report a multi-hospital nosocomial outbreak of fungemias due to this agent. Patients with positive blood culture to this agent were studied after six bloodstream infections identified in three Chilean hospitals in July 2013 were reported to Ministry of Health National Infection and Prevention Control Program. In general, there were mild clinical manifestations, without deaths attributable to the infection. Epidemiological and microbiological study identified 65 cases in 8 hospitals, mostly pediatric patients in chemotherapy. Initial studies of 94 different drugs and medical devices had negative results, until a second analysis of specific blisters and their pharmaceutical matrix selected by epidemiological criteria identified an intrinsic contamination of ondansetron blisters from a specific producer used in all the patients. A recall of contaminated ondansetron blisters was performed in all the country, after which the outbreak was contained. Surveillance and response of local and national infection prevention and control programs and laboratory support were key to control of a national multi-hospital common source outbreak due to contamination of a drug by an unusual fungus.

Key words: Bloodstream infections; fungal outbreak; Sarocladium kiliense; Acremonium; fungemia; intrinsic contamination.

Palabras clave: Infección del torrente sanguíneo; brote de infecciones fúngicas; Sarocladium kiliense; Acremonium; fungemia; contaminación intrínseca.

\section{Introducción}

S arocladium kiliense, antes conocido como Acremonium kiliense ${ }^{1}$, es un hongo saprófito de ubicación ubicua en el ambiente, ocasionalmente involucrado como agente oportunista en infecciones en humanos con compromiso inmunitario ${ }^{2}$ y responsable de micetomas e infecciones oculares secundarias a lesiones penetrantes en países tropicales ${ }^{3}$. Si bien los reportes de infecciones por este agente son limitados, se ha observado aumento en las infecciones causadas por éste, asociado a condiciones capaces de alterar el sistema inmunológico y procedimientos médicos invasores con fines diagnósticos y terapéuticos ${ }^{4}$ tales como recambios valvulares cardiacos, diálisis peritoneal, neurocirugía, catéter venoso central (CVC) y trasplante renal ${ }^{3,5-7}$. También se ha asociado a brotes nosocomiales, con pequeña magnitud, de endoftalmitis, en pacientes sometidos a cirugía de cataratas ${ }^{8} \mathrm{y}$ fungemias asociadas a CVC en pacientes oncológicos ${ }^{9}$, todos restringidos a un establecimiento y ninguno por contaminación intrínseca de productos farmacológicos.
En Chile, existe desde 1983 en el Ministerio de Salud (MINSAL) un Programa Nacional de Control de Infecciones Asociadas a la Atención de Salud (IAAS) caracterizado por la implementación en todos los hospitales del país, de un equipo profesional capacitado en IAAS, la formación y capacitación del personal de salud, vigilancia epidemiológica, regulación basada en evidencia y evaluación externa periódica de los hospitales.

Se presenta a continuación el reporte de un brote multicéntrico de fungemia por $S$. kiliense asociado a la contaminación intrínseca de ondansetrón intravenoso en pacientes inmunocomprometidos.

\section{Métodos}

\section{Antecedentes}

En julio de 2013, semana epidemiológica 28 (s28), el Programa Nacional de Control de IAAS (PNCI) del MINSAL recibió una comunicación desde el Programa de IAAS de un hospital pediátrico (hospital A) por la
IPrograma de Control de Infecciones Asociadas a la Atención de Salud, Departamento de Calidad y Seguridad de la Atención, Subsecretaría de Redes Asistenciales, Ministerio de Salud de Chile.

2Ex integrante del Departamento de Calidad y Seguridad del Paciente, Subsecretaría de Redes Asistenciales, Ministerio de Salud Chile.

${ }^{3}$ Agencia Nacional de Medicamentos, Instituto de Salud Pública de Chile.

${ }^{4}$ Ex Miembro de Departamento Biomédico, Instituto de Salud Pública de Chile.

5Laboratorio de Micología, Instituto de Salud Pública de Chile.

${ }^{6}$ Programa de Control de Infecciones Asociadas a la Atención de Salud, Hospital Dr. Luis Calvo Mackenna.

${ }^{7}$ Sub Departamento de Genética Molecular, Instituto de Salud Pública de Chile.

${ }^{8}$ Microbiología de Alimentos y Agua, Instituto de Salud Pública de Chile.

${ }^{9}$ Subdepartamento de Enfermedades Infecciosas, Instituto de Salud Pública de Chile.

Fuente de financiamiento: no hubo.

Recibido: 22 de diciembre de 2017

Aceptado: 14 de junio de 2018

Correspondencia a: Mauro Orsini Brignole mauro.orsini@minsal.c 
inusual identificación de tres casos, en dos semanas, de fungemias asociadas a CVC en pacientes oncológicos por Acremonium spp. Concomitantemente se tomó conocimiento de tres casos similares en pacientes oncológicos en otros dos hospitales pediátricos (hospital B, 2 casos y hospital C, 1 caso) observados entre las semanas epidemiológicas 26 y 28 de 2013. Ante estas notificaciones se planteó el diagnóstico de un brote multicéntrico de IAAS por Acremonium spp. Para su estudio, el MINSAL formó un grupo multidisciplinario que incluyó epidemiólogos, microbiólogos, médicos clínicos, personal de control de infecciones de los hospitales involucrados e instituciones tales como el laboratorio de referencia nacional (Instituto de Salud Pública- ISP) y el sistema nacional de compra centralizada de medicamentos de Chile (Central Nacional de Abastecimiento o CENABAST).

\section{Descripción de los casos iniciales}

El análisis de estos primeros seis casos permitió identificar que se trataba de pacientes pediátricos entre 3 y 14 años, todos con enfermedades neoplásicas (leucemia, osteosarcoma, neuroblastoma) en tratamiento y con uso de dispositivos vasculares centrales. Todos los casos se encontraban vivos y los cuadros clínicos eran leves e inespecíficos, observándose principalmente la presencia de fiebre y taquicardia (Tabla 1).

\section{Investigación inicial, definiciones y estudio epidemiológico (Figura 1)}

Considerando la descripción inicial de los casos, se estableció como hipótesis principal la contaminación de un fármaco intravenoso utilizado en pacientes oncológicos y como hipótesis alternativa, la contaminación de algún insumo con acceso intravascular de uso común en estos pacientes. Para el estudio inicial, se definió como caso a "un paciente con o sin síntomas, con identificación de Acremonium spp. en un hemocultivo" y como sospechoso a "un paciente con identificación de un hongo filamentoso en la sangre, independientemente del método de identificación utilizado".

Tabla 1. Descripción de los primeros seis casos identificados

\begin{tabular}{ccccl}
\hline Caso & Hospital & Sexo & Edad & Diagnóstico \\
\hline 1 & B & Masculino & 6 años & Leucemia linfoblástica aguda \\
\hline 2 & A & Masculino & 11 años & Osteosarcoma \\
\hline 3 & A & Femenino & 8 años & Leucemia linfoblástica aguda \\
4 & A & Femenino & 14 años & Leucemia linfoblástica aguda \\
\hline 5 & B & Femenino & 3 años & Neuroblastoma \\
6 & C & Masculino & 3 años & Neuroblastoma \\
\hline
\end{tabular}

En conjunto con los equipos de los hospitales se elaboró un listado inicial de los insumos y soluciones de administración intravascular utilizados por los casos para identificar los de uso común, comparando marcas comerciales y origen de los productos. Se comprobó en terreno que la reconstitución de fármacos oncológicos no era compartida por los establecimientos, descartándose que se tratara de contaminación durante este proceso y se planteó la posibilidad del uso de fármacos, soluciones o insumos contaminados intrínsecamente. Con esta hipótesis, se enviaron a estudio microbiológico al ISP muestras de los productos utilizados coincidentemente en al menos cinco de los pacientes, incluyéndose 35 muestras de medicamentos (ondansetrón, heparina, corticoesteroides), 44 muestras de soluciones parenterales (soluciones de potasio y cloruro de sodio) y 15 muestras de dispositivos médicos (catéteres, jeringas y otros), así como muestras ambientales (superficies y aguas) de las áreas de preparación de fármacos oncológicos, obteniéndose resultados negativos en todas.

Ante estos resultados, se envió desde MINSAL a todos los hospitales del país la instrucción de reportar cualquier resultado de hemocultivo con hongos filamentosos y enviarlo inmediatamente a estudio al ISP. Simultáneamente, se designó un equipo profesional para aplicar una encuesta epidemiológica a cada uno de los casos. Durante esta etapa, el ISP reclasificó el agente como Sarocladium kiliense.

El año 2013 finalizó con la identificación de 40 casos en 37 pacientes (tres pacientes tuvieron más de un episodio de fungemia), provenientes de cinco establecimientos, predominando pacientes oncológicos pediátricos; además, dos casos pediátricos con enfermedad inflamatoria intestinal y un caso en un paciente adulto de 43 años, con cáncer.

Las tasas de ataque de los hospitales con casos identificados variaron entre $0,05 \%$ y $6,2 \%$, con una mediana de $0,7 \%$, considerando como expuestos a los pacientes con quimioterapia. Al considerar sólo los hospitales pediátricos, la tasa de ataque primaria fue de 3,3\% (34 casos en 1.044 pacientes con quimioterapia).

A partir de enero de 2014 se modificó la definición de caso, utilizándose como caso sospechoso "paciente con identificación de hongo filamentoso en un hemocultivo" y como caso confirmado "paciente con identificación de Sarocladium kiliense en un hemocultivo, con estudio genético confirmatorio por el Subdepartamento de Genética Molecular y el Laboratorio de Referencia de Micología del ISP".

Como durante el análisis de los productos administrados se observó la ausencia de registros de marcas y lotes de los insumos y fármacos administrados individualmente a cada paciente, se consultaron los registros de stock de las farmacias de los establecimientos con casos identificados en el período estudiado. Para estos fines, así como el estudio microbiológico de fármacos, insumos y 


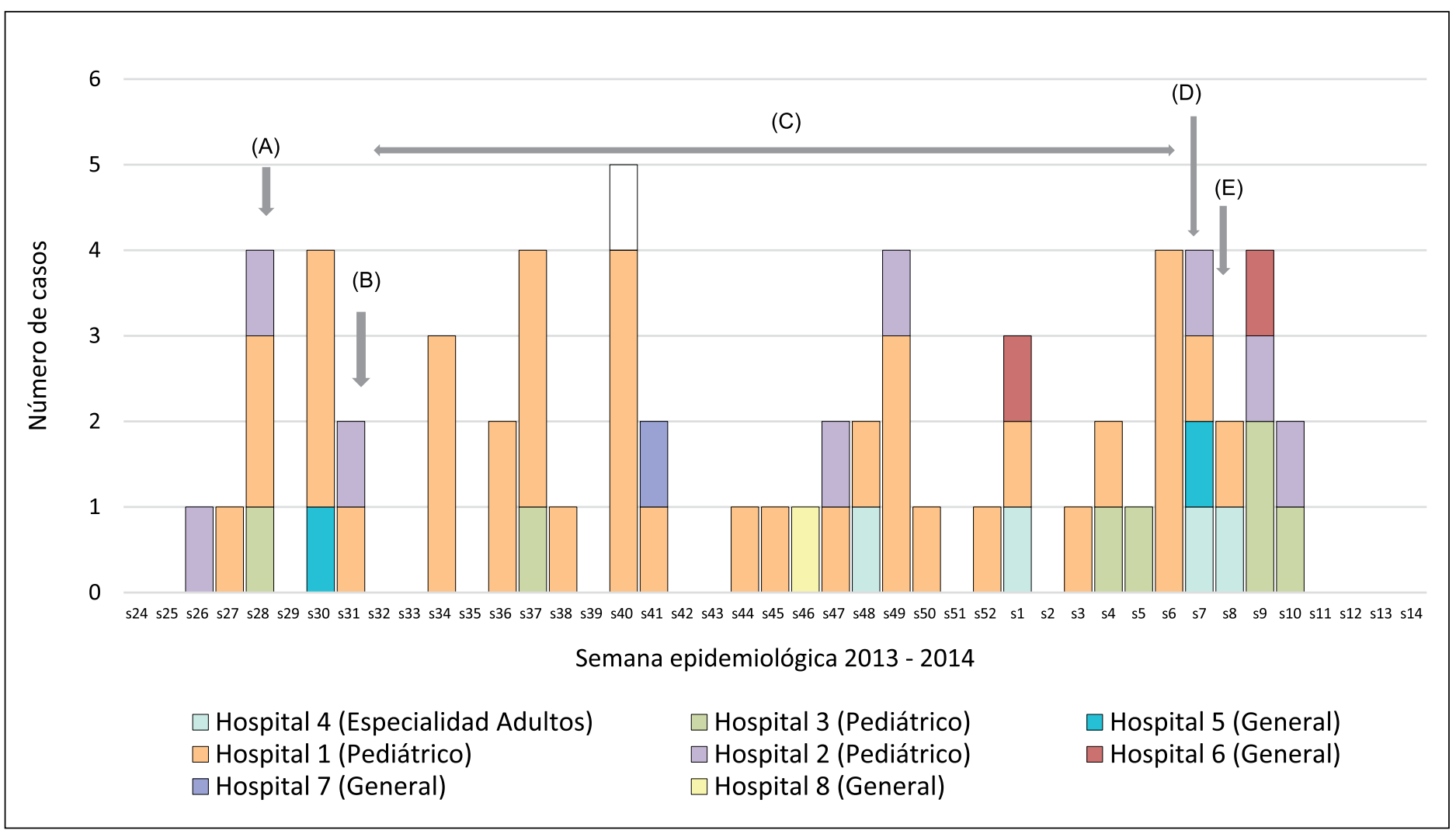

Figura 1. Curva epidémica de casos fungemia por Sarocladium kiliense por fecha de toma de primer cultivo positivo, Chile 2013-2014. Curva Epidémica: (A) reporte de caso índice; (B) alerta a hospitales con instrucciones de notificar casos; (C) estudio epidemiológico; fiscalización de sitios de producción de quimioterapia; recopilación y estudio de 4.685 muestras de fármacos, soluciones e insumos; (D) identificación de contaminación del lote de ondansetrón y retiro desde los establecimientos de salud; (E) implementación de protocolos de seguimiento de personas con catéter central + uso de ondansetrón y de tratamiento de casos.

soluciones identificadas como sospechosas, se designó a la Agencia Nacional de Medicamentos (ANAMED) del ISP como responsable.

\section{Descripción temporal de los casos}

Entre la semana epidemiológica 26 de 2013 y la semana 6 de 2014 se identificaron 53 casos en 50 pacientes, observándose $50,9 \%$ de los casos entre la semana 26 y la 40 de 2013, con un promedio de 1,5 casos semanales ( 0 a 5 casos semanales) (Figura 1).

\section{Descripción espacial de los casos}

Cuarenta y siete de los $53(88,7 \%)$ casos se identificaron en tres hospitales pediátricos, de los cuales uno concentró $69,8 \%$ del total (37 casos). El 11,3\% de los casos restantes se presentaron en cinco hospitales distintos, cuatro de ellos generales y uno de especialidad de adultos. Treinta y un casos $(58,5 \%$ del total) se identificaron en unidades de oncología y $12(22,6 \%)$ en servicios de urgencia, de los cuales $11(91,6 \%)$ tenían antecedente de hospitalización en unidades de oncología y concurrieron llamados por el equipo clínico como consecuencia de la alerta epidemiológica o por presentar fiebre u otro síntoma.

\section{Descripción clínica de los casos}

Se obtuvo los antecedentes clínicos de 52 de 53 casos de fungemia observadas en 49 pacientes, con igual proporción de sexo masculino y femenino. El 96,1\% tuvo un diagnóstico oncológico $(50,0 \%$ leucemia o linfomas y $46,1 \%$ tumores sólidos). El $90 \%$ de los casos tenía 16 años o menos al momento del diagnóstico (edad promedio 10,2 años $\pm 6,9$ años, rango 4 meses a 42 años). La letalidad cruda observada fue de $3,8 \%$ ( 2 casos), sin identificarse asociación a la infección en ninguno de ellos de acuerdo con la auditoría local (Tabla 2).

Los 52 casos contaban con acceso venoso central, de los cuales $75,0 \%$ eran catéteres con reservorio, y $20,3 \%$ transitorio, existiendo dos catéteres simultáneos en 4,7\% de los casos. No hubo una misma marca o modelo de catéter en todos, descartándose la hipótesis de contaminación de un tipo de catéter. En promedio, los casos 


\begin{tabular}{|lcc|}
\hline Tabla 2. Patología de base de $\mathbf{5 2}$ casos con fungemia por Sarocladium & killiense \\
\hline Diagnóstico principal & $\mathbf{n}$ & $\%$ \\
\hline Tumores sólidos & 24 & 46,1 \\
\hline Osteosarcoma & 12 & 23,0 \\
\hline Neuroblastoma & 3 & 5,8 \\
\hline Sarcoma sinovial & 3 & 5,8 \\
\hline Rabdomiosarcoma & 2 & 3,8 \\
\hline Coriocarcinoma embrionario & 1 & 1,9 \\
\hline Hepatoblastoma & 1 & 1,9 \\
\hline Sarcoma de Ewing & 1 & 1,9 \\
\hline Teratoma inmaduro de ovario & 1 & 1,9 \\
\hline Leucemia aguda & 21 & 40,4 \\
\hline Leucemia linfoblástica aguda & 13 & 25 \\
\hline Leucemia linfoblástica aguda & 6 & 11,5 \\
\hline Leucemia mieloide aguda & 2 & 3,9 \\
\hline Linfomas & 4 & 7,7 \\
\hline Linfoma linfoblástico mediastínico & 1 & 1,9 \\
\hline Linfoma de Burkitt & 1 & 1,9 \\
\hline Linfoma de Hodgkin & 1 & 1,9 \\
\hline Linfoma de células germinales anaplásico & 1 & 1,9 \\
\hline Leucemia crónica linaje ambiguo & 1 & 1,9 \\
\hline Enfermedad de Crohn & 2 & 3,9 \\
\hline Total & 52 & 100 \\
\hline
\end{tabular}

llevaban 233,5 días de uso de catéter con reservorio hasta el momento del cultivo (rango 3-2.348 días), la mitad de ellos con al menos 108 días de uso. En los casos con catéter transitorio, el promedio fue de 21,8 días de uso, en la mitad de los casos con al menos 18 días de uso (rango 3-371 días).

En 49 casos $(92,3 \%$ del total), la solicitud de hemocultivo se realizó por la presencia de un cuadro clínico inespecífico subyacente, describiéndose como los signos

Tabla 4. Principales hallazgos de laboratorio al momento de solicitud de hemocultivos en los $\mathbf{5 2}$ casos con fungemia por Sarocladium killiense

$\begin{array}{lcc}\text { Hallazgo } & \begin{array}{c}\text { Número de casos } \\ \text { con información }\end{array} & \begin{array}{c}\text { Mediana } \\ \text { (rango) }\end{array} \\ \text { Edad al momento de obtener hemocultivo (años) } & 52 & 9,7(0,3-42,6) \\ \text { Días entre hospitalización y cultivo } & 49 & 2,0(0-365) \\ \text { Último* hematocrito (\%) } & 50 & 28,0(16-39) \\ \text { Última*hemoglobina (g/dL) } & 50 & 9,5(5-13) \\ \text { Último* Recuento leucocitos/mm } & 48 & 3.180(30-13.003) \\ \text { Último* recuento absoluto de neutrófilos } & 31 & 1.296(0-21.663) \\ \text { Última* proteína C reactiva (mg/L) } & 30 & 24,0(3-195) \\ \text { * en los } 7 \text { días previos al día de toma del primer hemocultivo positivo. } & \end{array}$

Tabla 3. Signos y síntomas presentes al momento de solicitud de hemocultivos en los $\mathbf{5 2}$ casos con fungemia por Sarocladium killiense

\begin{tabular}{lcc}
\hline Signos y síntomas & n & Total \\
\hline Fiebre & 45 & $86,5 \%$ \\
\hline Taquicardia & 44 & $84,6 \%$ \\
\hline Lesiones mucocutáneas & 23 & $44,2 \%$ \\
\hline Vómitos & 12 & $23,1 \%$ \\
\hline Náuseas & 7 & $13,5 \%$ \\
\hline Diarrea & 6 & $11,5 \%$ \\
\hline Hipotensión arterial & 3 & $5,8 \%$ \\
Asintomáticos & 3 & $5,8 \%$ \\
\hline
\end{tabular}

más frecuentes la presencia de fiebre y taquicardia (Tabla 3), y los hallazgos de laboratorio al momento del diagnóstico (Tabla 4).

Al momento de realizar el primer hemocultivo, los casos llevaban en promedio 16,3 días de hospitalización (rango 0 a 365 días). Los cultivos más utilizados fueron: los de acceso venoso central asociado a venoso periférico, solicitados en $55,8 \%$ de los casos, acceso venoso central asociado a venoso periférico y cultivo de punta de catéter, en $34,6 \%$ de los casos, acceso venoso central asociado a punta de catéter, en 5,8\% de los casos y sólo acceso venoso central en 3,8\% de los casos. El 86,5\% de los cultivos positivos se obtuvo desde hemocultivos a través de accesos venosos centrales y de accesos venosos centrales y periféricos ( 51,9 y $34,6 \%$, respectivamente), seguidos de hemocultivos de accesos venosos centrales asociados a periféricos y cultivo de punta de catéter $(5,8 \%$ de los casos), hemocultivos de accesos venosos centrales y cultivo de punta de catéter ( $3,9 \%$ de los casos) y hemocultivos periféricos o periféricos asociados a cultivo de punta de catéter ( $1,9 \%$ de los casos cada uno). Sobre el rendimiento de cada origen de muestra en el diagnóstico de los casos, el cultivo resultó positivo en $96,1 \%$ de los casos en los que se solicitara hemocultivo de acceso central (50 de 52 casos), 48,9\% de los casos en los que se solicitó hemocultivo periférico ( 23 de 47 casos) y $28,6 \%$ de los casos en los que se cultivó la punta del catéter (6 de 21 casos).

\section{Identificación del reservorio}

Con la nueva estrategia de investigación, se identificó en febrero de 2014 el uso de cuatro medicamentos comunes: ondansetrón $8 \mathrm{mg} / 4 \mathrm{~mL}$ (laboratorio 1), heparina $25.000 \mathrm{U} / 5 \mathrm{ml}$ (laboratorio 2), cloruro de sodio $0,9 \%$ ampolla $20 \mathrm{ml}$ (laboratorio 2) y cloruro de potasio $10 \%$ ampolla $10 \mathrm{ml}$ (laboratorio 2), instruyéndose la prohibición nacional del uso de los lotes existentes de estos fármacos y su custodia en los propios establecimientos. 


\section{Estudio microbiológico}

Análisis de laboratorio de ondansetrón $8 \mathrm{mg} / 4 \mathrm{~mL}$ (laboratorio 1): Dado que uno de los hospitales concentraba $69,8 \%$ de los casos, funcionarios de ANAMED retiraron de éste la totalidad de los cuatro productos farmacéuticos identificados (4.865 ampollas) para ser estudiados en el Laboratorio Oficial de Control de Medicamentos del ISP. En febrero de 2014, en cada uno de los lotes de estos cuatro productos farmacéuticos retirados, ANAMED practicó ensayos de esterilidad. Para esto se seleccionaron muestras de los productos sin abrir y se determinó el uso de pool de 30 a 40 ampollas de un mismo lote para aquellos productos contenidos en ampollas que tuvieran un volumen inferior a $\operatorname{los} 10 \mathrm{~mL}$, con el fin de aumentar la probabilidad de identificar el agente etiológico. Durante este estudio se identificó la presencia de contaminación por un hongo filamentoso en tres pool obtenidos a partir de lotes distintos del producto ondansetrón $8 \mathrm{mg} / 4 \mathrm{~mL}$ (laboratorio 1). Tras el cultivo de este hongo se identificó que, al igual que los aislados clínicos, presentaba características morfológicas compatibles con Sarocladium spp, confirmándose posteriormente que correspondía a S. kiliense.

Identificación morfológica: Todos los aislados fueron diagnosticados con base a sus características morfológicas y fisiológicas ${ }^{10,11}$. Los aislados clínicos fueron identificados como Sarocladium spp. por sus características morfológicas: hifas septadas, hialinas y delgadas, célula conidiógena monofialídica, conidios elipsoidales a cilíndricos dispuestos en cabezas mucosas, aunque estas características no siempre permiten identificar a nivel de especie a los miembros de este género ${ }^{3,12}$. No hubo desarrollo de clamidoconidias después de los 15 días en todos los cultivos.

Identificación molecular: La extracción de ADN se realizó en forma automática con la ayuda de Maxwell $16^{\circledR}$ siguiendo las instrucciones del fabricante. Se amplificó mediante reacción de polimerasa en cadena (RPC) una región de 600 pb de espaciador intergénico ubicado entre los genes ribosomales $18 \mathrm{~S}$ y $28 \mathrm{~S}$. Los productos amplificados fueron purificados y secuenciados en ambas direcciones en un equipo ABI 3500 de Applied Biosystems ${ }^{\circledR}$. Las secuencias obtenidas fueron ensambladas mediante los programas Chromas $^{\circledR}$ y Bioedit $^{\circledR}$ y comparadas con la secuencia de referencia de $S$. kiliense (JX499276). Todos los resultados fueron confirmados después por el Central Bureau voor Schimmelcultures de Países Bajos.

Estudios genéticos: Un grupo de 17 aislados clínicos y dos procedentes de lotes diferentes de ondansetrón fueron enviados a los Centros para el Control y Prevención de Enfermedades (CDC) de Atlanta, Estados Unidos de
América (E.U.A.), con el objetivo de investigar si existía una relación filogénica entre éstas, identificándose una alta homogeneidad de la secuenciación genómica realizada, sugiriendo fuertemente un origen común de las muestras estudiadas $^{13}$.

Estudios de suceptibilidad in vitro a los antifúngicos: Se realizaron pruebas de sensibilidad a los antifúngicos en laboratorios de "Division of Foodborne, Waterborne and Environmental Diseases" de los CDC, por microdilución en caldo, siguiendo la metodología estandarizada por el Clinical and Laboratory Standards Institute (CLSI) Documento M38-A2 ${ }^{14}$. Se eligieron cinco aislados para el estudio de susceptibilidad. Todas las cepas tuvieron un patron de sensibilidad idéntico, con alta resistencia a los antifúngicos estudiados. Los valores de CIM para cada antifúngico fueron los siguientes: anfotericina 32 $\mu \mathrm{g} / \mathrm{ml}$; voriconazol, itraconazol y posaconazol $>16 \mu \mathrm{g} /$ $\mathrm{ml}$; flucitosina $<256 \mu \mathrm{g} / \mathrm{ml}$, anidulafungina $1 \mu \mathrm{g} / \mathrm{ml}$, caspofungina $0,06 \mu \mathrm{g} / \mathrm{ml}$ y micafungina $2 \mu \mathrm{g} / \mathrm{ml}$.

\section{Intervención definitiva}

El 14 de febrero de 2014 (semana epidemiológica 7 de 2014), el MINSAL y ANAMED instruyeron el retiro de los lotes involucrados de ondansetrón $8 \mathrm{mg} / 4 \mathrm{~mL}$ (laboratorio 1) de todos los establecimientos y farmacias del país, ampliándose esta instrucción a todas las series comercializadas del fármaco el 18 de febrero de 2014. Se originaron posteriormente distintas sanciones al laboratorio encargado de la importación del producto y la suspensión del registro sanitario y autorización de comercialización de acuerdo a lo establecido en el Reglamento del Sistema Nacional de Control de los Productos Farmacéuticos de Uso Humano ${ }^{15}$. Dado que el fármaco contaminado provenía de un laboratorio internacional, se dio la alerta a la Organización Panamericana de la Salud en el contexto del reglamento Sanitario Internacional vigente.

Se instruyó en todo el país, con un protocolo elaborado en conjunto con el ISP y la Sociedad Chilena de Infectología, la realización de hemocultivos en todo paciente con CVC de larga duración que hubiera recibido ondansetrón del laboratorio y lotes involucrados recomendándose, en caso de diagnosticarse fungemia por $S$. kiliense, el retiro del catéter y tratamiento del caso con anfotericina B o voriconazol durante 10 días, pudiendo prolongarse de acuerdo a la evolución clínica.

Desde la instrucción de retiro de los lotes se identificaron 12 casos adicionales entre las semanas epidemiológicas 7 y 10 de 2014 en los mismos establecimientos antes descritos, de los que uno era asintomático y 11 sintomáticos (11 con taquicardia y 8 con fiebre), todos con 
el antecedente de exposición a al menos uno de los lotes de ondansetrón $8 \mathrm{mg} / 4 \mathrm{~mL}$ (laboratorio 1) identificados (8 pacientes con exposición anterior y 4 con exposición posterior a la fecha de la indicación de retiro del medicamento) (Figura 1).

\section{Discusión}

Si bien se han descrito brotes de infecciones por hongos asociados a la contaminación intrínseca de fármacos, como el ocasionado por contaminación de inyecciones de metilprednisolona en E.U.A. el año $2012^{16,17} \mathrm{el} \mathrm{rol} \mathrm{de} \mathrm{los}$ hongos en este tipo de brotes es ocasional, asociándose a endoftalmitis ${ }^{17-19} \mathrm{e}$ infecciones intestinales ${ }^{20}$.

En este caso describimos un brote multicéntrico de fungemias producido por S. kiliense. El brote, con 65 casos de fungemia (62 pacientes afectados) en ocho establecimientos públicos pertenecientes al Sistema Nacional de Servicios de Salud de Chile (SNSS), se generó por la administración intravenosa de un fármaco antiemético contaminado de fábrica en pacientes susceptibles por sus patologías de base. Posteriormente se comprobó que la magnitud del brote se extendió a otros países de América Latina ${ }^{13}$ en los cuales también se produjo la distribución del fármaco contaminado.

A pesar del alto número de casos identificados, no se registraron fallecidos con letalidad causada por la infección y sólo en uno de los dos fallecidos la auditoría realizada por médicos locales estimó que la infección pudo contribuir al fallecimiento, sin ser la causa de ella. Esta situación pudo asociarse a una baja virulencia del hongo, a pesar de tratarse de una población mayoritaria con inmunocompromiso y a la alta resistencia encontrada a los tratamientos antifúngicos, reflejada en las elevadas CIMs a los distintos compuestos antifúngicos estudiados, resultado consistente con otras publicaciones ${ }^{12,21}$.

Si bien no se ha podido explicar el motivo por el cual sólo un establecimiento concentró $56,3 \%$ de los casos, en circunstancias que el ondansetrón es utilizado por otros establecimientos que tratan pacientes oncológicos, una hipótesis fue el uso predominante de los lotes contaminados del fármaco en este establecimiento en pacientes oncológicos. Esto, debido a la coexistencia de distintos mecanismos de adquisición por parte de los hospitales del SNSS (centralizada a un proveedor único versus descentralizada a distintos proveedores). Los lotes contaminados fueron adquiridos desde el sistema nacional de compra centralizada de medicamentos de Chile (CENABAST) a un mismo laboratorio y posteriormente distribuidos a los distintos establecimientos de la red, por lo que hospitales en los cuales predominó la compra centralizada por medio de CENABAST, pudieron tener mayor probabilidad de utilizar uno de los lotes contaminados respecto de aquéllos en los cuales predominó el mecanismo de compra des- centralizada a otros laboratorios. Esta hipótesis no pudo comprobarse por la falta de registro clínicos completos y de identificación de las marcas y lotes de los medicamentos utilizados en cada caso, pudiendo completarse con dificultad la trazabilidad de éstos sólo hasta las farmacias en la mayoría de los hospitales.

En general, son tres los principales mecanismos por los cuales se pueden generar infecciones asociadas al uso de fármacos: por la administración de productos contaminados durante su manufactura (contaminación intrínseca) o su almacenamiento y por errores durante la administración de éstos a los pacientes. Mientras las infecciones asociadas a este último mecanismo pueden controlarse con medidas de prevención de infecciones dependientes del personal de salud (cumplimiento de técnica aséptica y precauciones estándares, en particular no utilizando en varios pacientes medicamentos destinados a un solo uso), las relacionadas a las primeras dos causas suelen ser desafíos para la Salud Pública, por cuanto su prevención se asocia a rigurosidad en el cumplimiento y control de prácticas de manufactura y almacenamiento (regulación y fiscalización). Y, por su capacidad de generar brotes de infecciones en un número elevado de casos en uno o más servicios clínicos, hospitales o países, requieren de la existencia de sistemas de vigilancia epidemiológica sensibles y capaces de notificar de manera expedita cualquier sospecha de su existencia a las autoridades pertinentes.

Considerando que el estudio y control de estos brotes requieren, por lo general, de la participación de varios actores, cualquier demora en la sospecha, información a las autoridades o coordinación de los acciones de respuesta, puede tener consecuencias relevantes en evitar que la exposición de otros pacientes en riesgo ${ }^{16,22,23}$.

En este caso, varias condiciones permitieron la identificación y control de este brote multicéntrico, en particular la presencia del Programa Nacional de Control de IAAS con un sistema de vigilancia epidemiológica y equipos de control de IAAS conformados por personal clínico entrenado, la existencia de un sistema de estudio de productos farmacéuticos, así como de control de productos de uso clínico en toda la cadena de producción, distribución y uso ${ }^{16}$. Paralelamente, la presencia de apoyo microbiológico y un Laboratorio de Referencia Nacional para microbiología fue fundamental considerando que los criterios morfológicos utilizados en los hospitales no son suficientemente confiables para este hongo ${ }^{10}$ requiriéndose de la identificación molecular de todos los aislados recibidos para conocer la especie involucrada ${ }^{12}$. De fallar algunos de éstos, la identificación precoz de brotes multicéntricos que pudieran generarse es de vital relevancia, surgiendo en estos casos, la necesidad de contar con protocolos y mecanismos de acción específicos, conocidos y coordinados para cada uno de los actores involucrados en su estudio. 


\section{Principales lecciones aprendidas}

- El estudio de brotes, en particular de brotes multicéntricos, requiere de un grupo multidisciplinario al más alto nivel para liderar el estudio del brote, así como del trabajo de distintos actores que deben contar con roles y responsabilidades bien definidas, en particular si se considera la institucionalidad del sistema de salud chileno y la posibilidad de los brotes de afectar tanto establecimientos públicos como privados.

- El estudio de brotes asociados a contaminación de productos farmacéuticos corresponde a un desafío de Salud Pública. Por los mismo, se requiere prioritariamente de protocolos estandarizados que respondan a algunas de las principales preguntas que se plantearon en este brote, tanto del punto de vista epidemiológico (número y criterio de selección de muestras a tomar y analizar e ésta) como microbiológico (metodología de cultivo y tipos de estudios a realizar).

\section{Resumen}

Introducción: Sarocladium kiliense es un hongo saprófito que puede generar infecciones oportunistas asociadas a procedimientos invasores. Se informa un brote multicéntrico nosocomial de fungemias de fuente común por este agente. Luego del reporte de cinco casos en pacientes en tres hospitales al Programa de Control de Infecciones del Ministerio de Salud de Chile en julio de 2013, se estudiaron a nivel nacional todos los pacientes con hemocultivo positivo para este agente. Se trató de cuadros clínicos leves a moderados, sin muertes atribuibles. El estudio identificó 65 casos en 8 hospitales, en su mayoría pacientes pediátricos en quimioterapia. Estudios iniciales de 94 muestras de cuatro fármacos y dispositivos usados en todos los casos resultaron negativas hasta que, en un segundo análisis de lotes seleccionados por criterios epidemiológicos y su matriz farmacéutica, se identificó la contaminación intrínseca de ampollas de ondansetrón de un productor específico, que se usó en todos los casos. Se realizó un retiro nacional de las ampollas de los tres lotes contaminados del fármaco, después de lo cual se contuvo el brote. La vigilancia de infecciones en los hospitales y el programa nacional coordinado con los laboratorios de microbiología fueron claves para identificar un brote multicéntrico de fuente común por contaminación de un fármaco por un hongo inusual.

\section{Referencias bibliográficas}

1.- Summerbell R C, Gueidan C, Schroers H J, de Hoog G S, Starink M, Rosete A Y, et al. Acremonium phylogenetic overview and revision of Gliomastix, Sarocladium, and Trichothecium. Stud Mycol. 2011; 68: 139-62. doi: 10.3114/sim.2011.68.06.

2.- Fernández-Silva F, Capilla J, Mayayo E, Sutton D, Guarro J. In vitro evaluation of antifungal drug combinations against Sarocladium (Acremonium) kiliense, an opportunistic emergent fungus resistant to antifungal therapies. Antimicrob Agents Chemother 2014; 58 (2): 1259-60. doi: 10.1128/AAC.02131-13.

3.- Guarro J, Gams W, Pujol I, Gené J. Acremonium species: new emerging fungal opportunists-in vitro antifungal susceptibilities and review. Clin Infect Dis 1997; 25: 1222-9.

4.- Fincher R M, Fisher J F, Lovell R D, Newman C L, Espinel-Ingroff A, Shadomy $\mathrm{H}$ J. Infection due to the fungus Acremonium (cephalosporium). Medicine (Baltimore) 1991; 70 (6): 398-409.

5.- Pastorino A C, De Menezes U P, Marques H H D S, Vallada M G, Cappellozi V L, Carnide E $\mathrm{M} \mathrm{G}$, et al. Acremonium kiliense infection in a child with chronic granulomatous disease. Braz J Infect Dis. 2005; 9 (6): 529-34. DOI: /S141386702005000600014 .
6.- Díaz-Couselo F, Zylberman M. Catheter-related Acremonium kiliense fungemia in a patient with ulcerative colitis under treatment with infliximab. Case Rep Infect Dis 2011; 2011: 1-2. doi: 10.1155/2011/710740.

7.- Valson A T, Kakde S T, Mohanraj P, Basu G, Mohapatra A, Varughese S. Resolution of Acremonium kiliense subcutaneous abscess in a renal allograft recipient without antifunga therapy. Transpl Infect Dis 2014; 16 (5): 864-5. (Letter).

8.- Fridkin S K, Kremer F B, Bland L A, Padhye A, McNeil M M, Jarvis W R. Acremonium kiliense endophthalmitis that occurred after cataract extraction in an ambulatory surgical center and was traced to an environmental reservoir. Clin Infect Dis 1996; 22 (2): 222-7.

9.- Ioakimidou A, Vyzantiadis TA, Sakellari I, Arabatzis M, Smias C, Douka V, et al. An unusual cluster of Acremonium kiliense fungaemias in a haematopoietic cell transplantation unit. Diagn Microbiol Infect Dis. Elsevier Inc.; 2013; 75(3): 313-6. doi: 10.1016/j.diagmicrobio.2012.11.015

10.- Gams W. Cephalosporium-artige Schimmelpilze (Hyphomycetes). 1st ed. Fische G, editor. Stuttgart; 1971. 262 p.

11.- De Hoog G, Guarro J, Gené J, Figueras M. Atlas of Clinical Fungi. 4th ed. Ultecht: Centraalbureau voor Schimmelcultures; 2014.

12.- Perdomo H, Sutton D A, García D, Fothergill A
W, Cano J, Gené J, et al. Spectrum of clinically relevant Acremonium species in the United States. J Clin Microbiol 2011; 49 (1): 243-56. doi: 10.1128/JCM.00793-10.

13.- Etienne K A, Roe C C, Smith R M, Vallabhaneni S, Duarte C, Escandón P, et al. Whole-genome sequencing to determine origin of multinational outbreak of Sarocladium kiliense bloodstream infections. Emerg Infect Dis 2016; 22 (3): 476-81. doi: 10.3201/ eid2203.151193.

14.- Clinical and Laboratory Standards Institute (CLSI). Reference Method for Broth Dilution Antifungal Susceptibiliy Testing of Filamentous Fungi; Approved Standard. M38-A2. 2nd ed. Clinical and Laboratory Standards Institute (CLSI), editor. Wayne, Pennsylvania: Clinical and Laboratory Standards Institute (CLSI); 2008.

15.- Ministerio de Salud. Aprueba reglamento del sistema nacional de control de los productos farmacéuticos de uso humano. Decreto $\mathrm{N}^{\circ} 3$ Chile: Biblioteca del Congreso Nacional de Chile; 2011 p. 56.

16.- Smith R M, Schaefer M K, Kainer M A, Wise M, Finks J, Duwve J, et al. Fungal infections associated with contaminated methylprednisolone injections. N Engl J Med 2013; 369 (17): 1598-609. doi: 10.1056/ NEJMoa1213978.

17.- Centers for Disease Control and Prevention 
(CDC). Notes from the field: Multistate outbreak of postprocedural fungal endophthalmitis associated with a single compounding pharmacy - United States, March-April 2012. MMWR Morb Mortal Wkly Rep 2012; 61 (17): 310-1.

18.- Cakir M, Imamoğlu S, Cekiç O, Bozkurt E, Alagöz N, Oksüz L, et al. An outbreak of early-onset endophthalmitis caused by Fusarium species following cataract surgery. Curr Eye Res 2009; 34 (11): 988-95. https://doi. org/10.3109/02713680903254822.

19.- Small K W, Chan C K, Silva-García R, Walsh T J. Onset of an outbreak of Bipolaris hawaiiensis fungal endophthalmitis after intravitreal injections of triamcinolone Ophthalmol 2014; 121 (4): 952-8. doi: 10.1016/j.ophtha.2013.10.040

20.- Cheng V C C, Chan J F W, Ngan A H Y, To K K W, Leung S Y, Tsoi H W, et al. Outbreak of intestinal infection due to Rhizopus microsporus. J Clin Microbiol 2009; 47 (9): 2834-43. doi: 10.1128/ JCM.00908-09.

21.- Fernández-Silva F, Capilla J, Mayayo E, Sutton DA, Hernández P, Guarro J. Evaluation of the efficacies of amphotericin $b$, posaconazole, voriconazole, and anidulafungin in a murine disseminated infection by the emerging opportunistic fungus Sarocladium
(Acremonium) kiliense. Antimicrob Agents Chemother 2013; 57(12): 6265-9. doi: 10.1128/AAC.01484-13.

22.- Vaux S, Criscuolo A, Desnos-Ollivier M, Diancourt L, Tarnaud C, Vandenbogaert M, et al. Multicenter outbreak of infections by Saprochaete clavata, an unrecognized opportunistic fungal pathogen. MBio 2014; 5 (6): 1-10. doi: 10.1128/mBio.02309-14.

23.- Vonberg R-P, Gastmeier P. Hospital-acquired infections related to contaminated substances. J Hosp Infect [Internet] 2007; 65 (1): 15-23. Available from: http://www.ncbi.nlm.nih. gov/pubmed/17145102. DOI: $10.1016 / \mathrm{j}$. jhin.2006.09.018. 